\title{
Wir müssen über einen neuen Ärzteeid diskutieren
}

\author{
Jean-Pierre Wils, Ruth Baumann, Bernhard Egger, Max Giger, Claudia Käch \\ «Eidkommission» der Stiftung Dialog Ethik
}

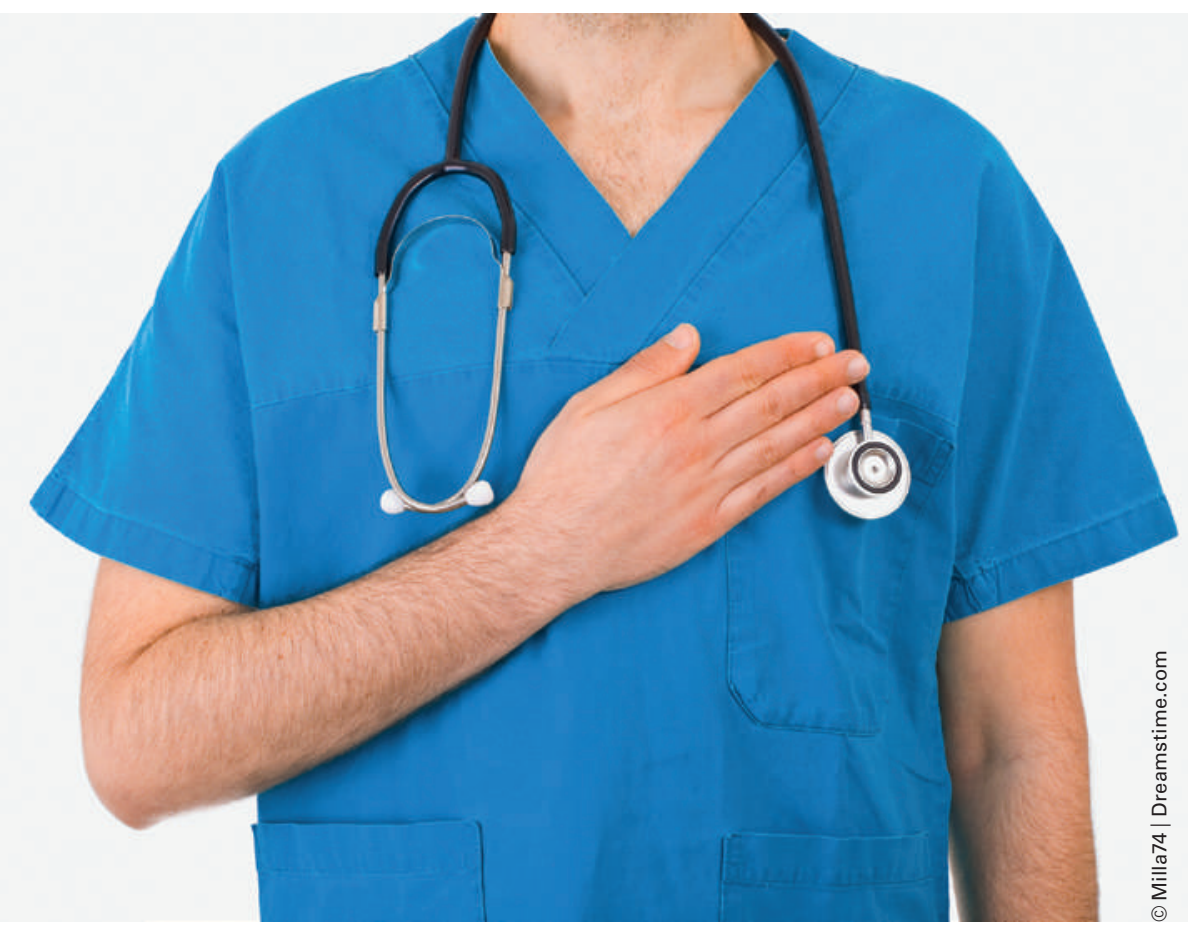

Braucht es einen neuen Ärzteeid? Die Meinungen gehen weit auseinander.

«Zeit für einen neuen Eid» übertitelte die Wochenzeitung DIE ZEIT am 22. November 2015 einen Artikel über Ärzteeide. An 17 von insgesamt 37 deutschen medizinischen Hochschulen legen die Absolventen auf eigenen Wunsch einen Eid ab. Ein Eid für den Arztberuf ist keineswegs ein Griff in den verstaubten Bestand der Medizingeschichte. Der Präsident der Bundesärztekammer unterstützt das Ansinnen: Ein Eid würde «der Profanisierung unseres Berufsbildes entgegenwirken». Die Generalversammlung des Weltärztebundes beauftragte im Oktober 2015 eine Arbeitsgruppe, abzuklären, welche Details des Genfer Ärztegelöbnisses den Zeitumständen angepasst werden sollen.

\section{Das Eid-Modell}

Genau diese Frage hat unser Eid-Modell ernst genommen, und die Antwortet lautet: Eine neue Eidformel ist dringend erforderlich, vor allem wegen des zunehmen- den ökonomischen Drucks, dem der Arztberuf ausgesetzt ist. Zu Recht schreibt Brigitte Muff: «Diese zunehmende Ökonomisierung in der Medizin ist nicht nur bedauernswert, sondern für die medizinische Qualität unserer Spitäler auch gefährlich» [1]. Unser Eid-Modell soll die Diskussion in der Ärzteschaft anstossen. Der Text ist weder endgültig noch überarbeitungsresistent. Die Diskussion ist kein «Zweck an sich». Ein Eid muss implementiert werden. Er kann nur wirksam sein, sobald er Bestandteil der beruflichen Praxis geworden ist.

\section{Beispiel: Business Schools}

Eide werden in anderen Berufen nicht nur diskutiert, sondern abgelegt: Der australische Ethiker Peter Singer wies im Artikel «After VW: Ethical business and the question of honesty» [2] darauf hin, dass die Studierenden der Harvard Business School 2009 einen Eid abgelegt hatten, in dem sie versprachen, dass sie ihre Arbeit zum Wohle aller Beteiligten und Betroffenen verrichten wollten. Seitdem legen Absolventen von 250 «Business Schools» einen Eid ab. Australien verfügt über einen «Banking and Finance Oath». 2015 haben sich in den Niederlanden 90000 Banker mittels eines Eides auf Integrität verpflichtet. Immer steht der potentielle oder reale Konflikt zwischen den Diktaten ökonomischen Profitstrebens und den Erfordernissen des Berufsethos im Vordergrund. Wer heute über Eide diskutiert, befindet sich in der Vorhut der Entwicklung.

Seit 2002 hat vor allem die Publikation Medical Professionalism in the new Millennium: A Physician Charter die Diskussion geprägt. Europäischen und amerikanischen Fachgesellschaften dient sie zur berufsethischen Orientierung. Bislang wurde jedoch kaum über einen praktikablen Eid diskutiert, der sich den Erfordernissen des medizinischen Alltags stellt. Diese werden zunehmend geprägt von Faktoren ökonomischer Provenienz. Was hat Priorität: das Ethos ärztlichen Handelns oder finanzielle Endresultate? In Deutschland gehen $46 \%$ der befragten Chefärzte davon aus, dass sie aus ökonomischen Gründen Patienten nützliche Massnahmen vorenthalten oder durch weniger effektive, weil kostengünstigere, ersetzt haben [3]. 


\section{Antworten auf Leserbriefe und Kommentare}

Dass der Eid geeignet sei, «der schleichenden Demontage des Arztberufs und der Resignation in den eigenen Reihen entgegenzuwirken", hebt Alfred Hunziker [4] hervor und trifft damit das Motiv, das hinter dem Eid-Modell steht, äusserst genau. In der gleichen Ausgabe der Schwei-

\section{Was hat Priorität: das Ethos ärztlichen Handelns oder finanzielle Endresultate?}

zerischen Ärztezeitung schreibt Walter Grete, dass das Eid-Modell "geeignet [sei], auch Eingang in den Weltärztebund zu finden». Selbstverständlich kann man dies als Zielgrösse betrachten, aber ohne eine wirksame Unterstützung aus Kantonal- und Fachverbänden kann ein solcher Weg nicht zurückgelegt werden. Die Einführung des neuen Ärzteeides wird von der Plenarversammlung der fmCh unterstützt. Der Vorstand der Schweizerischen Gesellschaft für Psychiatrie und Psychotherapie kann hinter den meisten Formulierungen des Eid-Modells stehen, ortet Probleme bei dessen Implementierung.

\section{Sinnentleerte Zeremonien?}

Bernhard Gurtner konfrontiert den Eid mit dem Verdacht, «zu sinnentleerten Zeremonien [zu] verkommen» [5]. Dem widersprechen die oben erwähnte Praxis der Eidablegung und das Wesen eines Eides. Eide sind feierliche Selbstverpflichtungen: Die Feierlichkeit ist ein wesentlicher Bestandteil des Wirksamwerdens der Verpflichtung und keinesfalls dekorativ oder äusserlich. Der Eid enthält keineswegs «nicht einlösbare Versprechen». Seine Bestandteile skizzieren das Ethos des medizinischen Alltags und verlangen an keiner Stelle aussergewöhnliche moralische Leistungen.

Die Beobachtung, die in der folgenden Frage enthalten ist, «Wer denn verhält sich im beruflichen Alltag immer freundlich und gesprächsbereit?», dokumentiert nicht das Unhaltbare eines Eides, sondern das Erfordernis, elementare Standards hervorzuheben, auch wenn sie nicht «immer» eingehalten werden. Das Argument, auch Hippokrates hätte das Arztgeheimnis «genauso verletzt", mutet seltsam an, als würden wir geradezu deshalb keine Normen brauchen, weil sie immer wieder verletzt werden. Walter Dinner behauptet zu Unrecht in der gleichen Ausgabe, dass «ethisches Verhalten durch einen offiziellen Eid um kein Haar besser oder schlechter» werde. Offenbar muss auch hier die faktische Abweichung von Normstandards als Argument für deren Überflüssigkeit herhalten. Dass eine Eidablegung «ein Akt» sei, «der letztlich lediglich eine Pseudogarantie für eine Verhaltensweise vorgau- kelt», ist als Argument nicht nachzuvollziehen. Eide waren noch nie Garantien und waren auch niemals so gedacht, also können sie auch keine "Pseudogarantie» bilden.

\section{Der Eid als Selbstkontrolle}

Ökonomisches Denken und Handeln korrumpieren Hausärzte, die bei Überweisungen der Patienten Kickbacks annehmen [6]. Überraschend stösst deshalb die Kritik der Gruppe Kielholz auf [7], die den Eid als «redundantes, pathetisches, fast schon religiös klingendes System von moralischen Verpflichtungen" ablehnt. «Schwierigen Güterabwägungen» im medizinischen Alltag werde er ohnehin nicht gerecht. Dass das EidModell religiöse Anklänge habe, entzieht sich unserer Wahrnehmung. Selbstverständlich können "schwierige Güterabwägungen" nicht durch Eide allein geleistet werden, denn ein Berufseid bezieht sich auf Haltungen bzw. Tugenden. Solche Abwägungen geschehen gleichwohl in einem Raum, der geprägt ist von einem klaren Ethos. Fehlt ein solches, werden die Abwägungen nicht einfacher bzw. vertrauenswürdiger. Der Gruppe entgeht offenbar, dass Eide nicht zu den von ihr aufgezählten externen Kontrollinstanzen gehören, sondern eine wirksame Form der Selbstkontrolle darstellen und somit ein Mittel zu Sicherung von Autonomie sind. Zu Recht schreibt Werner Saameli [8]: «Vielleicht braucht es neben der Standesordnung und den Richtlinien der SAMW eben doch einen zusätzlichen Weg, um die berechtigten Anliegen durchzusetzen.»

\section{Was sagt die Medizinethik?}

Rouven Porz vermittelt in seinem Beitrag [9] Wissenswertes über die Trennung der «eigenen Moralvorstellungen von den Ansprüchen der Berufsrolle» und geht auch auf das Eid-Modell ein. Er fragt sich, ob angesichts der "Ökonomisierung der Medizin die einfache Formel, dass die Patientin doch immer im Fokus aller Bemühungen stehen muss» weiterhelfe. Wer sagt aber, dass sie nicht weiterhelfe? Darüber hinaus ist diese «einfache Formel» keineswegs einfach. Sie verlangt einiges und

\section{Leider hat die Medizinethik zum Thema des} Berufsethos ziemlich erfolgreich geschwiegen.

kann, wie der Eid zeigt, durchaus operationalisiert werden. Rouven Porz hebt die Wichtigkeit der Medizinethik hervor. Deren Konfrontation mit einem «Eid» mutet jedoch seltsam an: «Die ganze Medizinethik als Disziplin versucht dort Hilfe zu bieten, wo ein einfacher Eid nicht mehr ausreicht. Die Anforderungen an die Berufsrollen von (post)modernen Ärztinnen und Ärzten sind leider nicht mehr in trivialen Lebensregeln zu fassen.» 
Was "(post)moderne» Ärzte sind, ist uns ein Rätsel und deshalb bleibt dieses Prädikat als Argument düster. Offenbar verwechselt Rouven Porz die Bedeutung von «trivial» mit der von "einfach». Die Regeln des EidModells sind tatsächlich sprachlich einfach gehalten und sie sind «einfach» im Sinne von «elementar» und «wichtig». Deshalb sind sie aber noch längst nicht «trivial». Die Medizinethik als akademische Disziplin kümmert sich um schwierige, schwerwiegende, komplexe

\section{Wenn wir etwas versprechen, machen wir deutlich, dass das Versprochene für uns gilt.}

und umstrittene Sachverhalte in der Medizin. Diese aber bilden nicht den Alltag der Ärztin. Leider hat die Medizinethik zum Thema des Berufsethos ziemlich erfolgreich geschwiegen. Der blosse Rekurs auf die vier Prinzipien von Beauchamp und Childress, die von Rouven Porz genannt werden, hilft nicht weiter, denn sie kennzeichnet ein hoher Abstraktionsgrad.

\section{Pathos}

Erhard Taverna kritisiert mit dem schönen, aber unangemessenen Titel «Die Schwurhand» das Eid-Modell und wirft dem Eid «Pathos» vor [10]. Selber greift er schwer und sehr tief in die Pathos-Kiste, wenn er Eide wahlweise und wahllos mit «Gott», dem «Teufel», der «Verdammnis», dem «Strafgericht», gar «einem Führer» und einer «Fahne» assoziiert - und eben deshalb auf «die Schwurhand» reduziert. Dass "wir keine Priester mehr sind" können "wir» gerne zustimmen. Der Eid ist ein performativer Akt, wie ein ganz gewöhnliches Versprechen. Er stiftet die Verbindlichkeit, welche die in ihm enthaltenen Haltungen fordert. Dazu braucht es weder «Gott» noch «Teufel». Wenn wir etwas versprechen, machen wir deutlich, dass das Versprochene für uns gilt. Wir stellen uns unter die Gültigkeit des Versprochenen. Das tun wir genauso, indem wir einen Eid ablegen. Das hat mit «magischem Denken» nun wirklich nichts zu tun.

\section{Fazit}

Die Ärzteschaft steht wie kaum eine andere Berufsgruppe vor der Aufgabe, Wissen und hohes Können mit den Werten der Humanität zu verbinden. Das gehört zu ihrer Professionalität. Ihre «Tugenden» finden sich in einem eigenen Berufsethos wieder, in Haltungen, die zu der spezifischen Ehre und Würde des Berufs beitragen. Ärzte und Ärztinnen haben eine Schrittmacherfunktion in unserer Gesellschaft. Sie sollten ihre Denkweise nicht ökonomisieren lassen. Die Diskussion über einen verpflichtenden, aktualisierten Ärzteeid soll auf breiter Basis fortgeführt werden. Wir teilen die Meinung der Psychiaterinnen und Psychiater, dass sein Inhalt in die Weiter- und Fortbildung verbreitet werden soll.

\footnotetext{
Literatur

1 Muff B. Ökonomie verdrängt Hippokrates - der Wertewandel im Spital. Schweiz Ärztezeitung. 2016;97(5):187-90.

2 The Globe and Mail, 13.10.2015

3 Reifferscheid A, Pomorin N, Wasem J. Ausmass von Rationierung und Überversorgung in der stationären Versorgung. Dtsch med Wochenschr 2015; 140(13): e129-e135

4 Hunziker A. Anstoss zur Rückbesinnung. Schweiz Ärztezeitung. 2015;96(38):1372-3

5 Gurtner B. Eidgenossen und ziviler Ungehorsam. Schweiz Ärztezeitung. 2015;96(29-30):1052-3.

6 Diener M. Hausärzte: Patienten an den Meistbietenden. K-Tipp, 25.02.2015

7 Kielholz R, Möller A, Schmid PA et al. Ein Eid für die heutigen Ärztinnen und Ärzte? Schweiz Ärztezeitung. 2015; 96(27-28):1020

8 Saameli W. Es ist dringend nötig, Gegensteuer zu geben. Schweiz Ärztezeitung. 2015;96(31-32):1090

9 Porz R. Werte, Moralvorstellungen und Berufsrollen. Schweiz Ärztezeitung. 2015:96(42):1536

10 Taverna E. Die Schwurhand. Schweiz Ärztezeitung. 2015;96(2930):1078
} 